\title{
Retrieving new information on ichthyosis
}

Ichthyosis is a broad term for a group of hereditary skin disorders with various presentations, usually including scaling of the skin. Some forms are relatively mild, whereas others are quite severe. Previous research has identified several genes and gene regions that are associated with some forms of ichthyosis, but for other forms, the underlying genetic causes are not known. For rare diseases, determining the genetic causes can be challenging, in part because so few people are affected by them that researchers cannot obtain sufficient examples.

Animal models can be useful both for identifying causative mechanisms for disorders that aren't understood and for evaluating treatment options for disorders whose bases are known. For example, mutations in two genes (KRT10 and TGM1) are associated with forms of ichthyosis in both humans and dog breeds (Norfolk terrier and Jack Russell terrier, respectively). Because each dog breed has a high degree of homogeneity, they are promising models for studies of genetically linked diseases such as ichthyosis.
In 2007, a spontaneous ichthyosis resembling some forms of human ichthyosis was described in golden retriever dogs. Recently, a group of European researchers directed by Catherine André (Centre National de la Recherche Scientifique and Université Rennes 1, Rennes, France) and Judith Fischer (Centre National de Génotypage, Evry, France and Institute for Human Genetics and Universität Freiburg, Freiburg, Germany) carried out a genetic association study in 40 golden retrievers to look for potential causes of this ichthyosis. The team found that the ichthyosis in these dogs was caused by mutations in the gene PNPLA1. Furthermore, mutations in the human version of the gene were found in six individuals from two families with ichthyosis for which previous studies had failed to identify a cause (Nat. Genet. 44, 140-147; 2012).

The protein encoded by PNPLA1 belongs to a family of phospholipases, enzymes that break down fats, including those that form cell membranes. In skin samples

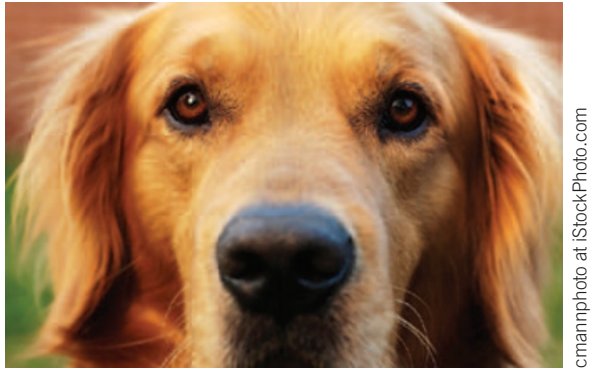

from golden retrievers and one person with ichthyosis and PNPLA1 mutations, membranes of cells in the epidermal skin layer were abnormal. PNPLA1 was not previously known to be associated with ichthyosis or other diseases; the discovery of its involvement provides a new link between the PNPLA gene family and ichthyosis.

Forms of ichthyosis occur in several other dog breeds, and these study results offer hope that similar genetic association studies in those breeds may help to identify other genes involved in human ichthyosis.

Monica Harrington

\section{TINY SPHERES DELIVER RNA TO FIGHT CANCER}

RNA interference (RNAi) is a naturally occurring process in cells that suppresses gene expression. Short interfering RNA (siRNA) binds to messenger RNA, which carries genetic information, and destroys it before it can be translated into protein. RNAi thereby provides a powerful tool for shutting off malfunctioning genes, and recent research has been focused on delivering siRNAs designed to target specific genes to treat diseases such as cancer.

A major challenge of developing treatment approaches based on RNAi is finding an efficient delivery system for the RNA. A team of researchers led by Paula T. Hammond (Massachusetts Institute of Technology, Cambridge) has searched for a way to tightly pack the short strands of siRNAs into nanoparticles small enough to make their way into target cells. "It's been a real struggle to try to design a delivery system that allows us to administer siRNA, especially if you want to target it to a specific part of the body," Hammond said in a press release.

Now, the team has found a way to take advantage of the DNA/RNA machinery provided by nature. Using a method called rolling circle transcription, they produced long, pure RNA strands that could assemble themselves into a delivery system by winding up into compact spheres. These small spheres, which they call RNAi-microsponges, are so dense that they withstand degradation until they reach their destination in the body, where the RNA structures are converted into siRNA that can actively silence genes (Nat. Mater. doi:10.1038/nmat3253; published online 26 February 2012).

The researchers tested the efficiency of their delivery system by designing RNA sequences targeting a gene that causes tumor cells to glow in mice. They delivered the highest number of siRNA molecular copies ever encapsulated in a nanoparticle, and the RNAi-microsponges significantly reduced expression of the targeted gene. Even with a smaller dose of particles, the new system could be used to knock down expression of specific genes as effectively as existing delivery methods.

These microsponges offer a way to treat cancer and other chronic diseases that may be caused by a faulty gene, without the negative effects of existing delivery methods. "RNA interference holds a huge amount of promise for a number of disorders, one of which is cancer, but also neurological disorders and immune disorders," Hammond said. Next, the researchers are working to develop DNA-carrying spheres, which may be used in gene therapy approaches. 